\title{
SOME CHARACTERS OF KAC AND WAKIMOTO AND NONHOLOMORPHIC MODULAR FUNCTIONS
}

\author{
KATHRIN BRINGMANN AND KEN ONO
}

\begin{abstract}
Answering a question of Kac, we relate the character formulas for certain $s \ell(m, 1)^{\wedge}$ modules to automorphic forms. We show that these $q$-series are the "holomorphic parts" of nonholomorphic modular functions.
\end{abstract}

\section{INTRODUCTION}

In an important series of papers [3, 4, 9], Kac and Wakimoto discovered deep connections between the representation theory of affine Lie superalgebras and number theory. Their 1994 paper [3] shed new light on the representation of integers as sums of squares, and it also inspired subsequent works in the subject by Milne, the second author, and Zagier $[5,6,7,10]$. In the same paper, Kac and Wakimoto also noticed that a $q$-series related to Ramanujan's mock theta functions is the denominator identity for the affine superalgebra $s \ell(2,1)^{\wedge}$. In his 2002 Ph.D. thesis, Zwegers [12] related Ramanujan's mock theta functions to weight $1 / 2$ harmonic Maass forms, thereby forging a connection between representation theory and harmonic Maass forms (at least in the case of $s \ell(2,1)^{\wedge}$ ).

In their 2001 paper [4], Kac and Wakimoto computed characters related to $g \ell(m, 1)^{\wedge}$ and $s \ell(m, 1)^{\wedge}$, and they represented them in terms of theta functions and a function they referred to as a "multivariable Appell function" (see $\S 4$ of [4]). In view of the recent works on harmonic Maass forms and Ramanujan's mock theta functions (for example, see $[8,11,12])$, Kac asked whether there is a connection between such characters and harmonic Maass forms. This question is of particular interest due to the fact that Kac and Peterson obtained the modularity of similar characters in earlier work [2].

Here we show that some of these $q$-series are (up to powers of $q$ ) indeed related to modular forms. They turn out to be the "holomorphic parts" of some very nice nonholomorphic modular functions. Moreover, these nonholomorphic modular functions turn out to be the product of a weight 1/2 harmonic Maass form with a weight $-1 / 2$ quotient of Dedekind eta-functions.

We consider the Kac-Wakimoto character formulas for the $\operatorname{s\ell }(m, 1)^{\wedge}$ modules $L\left(\Lambda_{(s)}\right)$, where $L\left(\Lambda_{(s)}\right)$ is the irreducible $s \ell(m, 1)^{\wedge}$ module with highest weight $\Lambda_{(s)}$ (see [4] for definitions and background). If $m \geq 2$ and $s \in \mathbb{Z}$, then their work implies that

$$
\operatorname{tr}_{L\left(\Lambda_{(s)}\right)} q^{L_{0}}=2 q^{-\frac{s}{2}} \cdot \frac{\phi\left(q^{2}\right)^{2}}{\phi(q)^{m+2}} \cdot \sum_{k=\left(k_{1}, k_{2}, \ldots, k_{m-1}\right) \in \mathbb{Z}^{m-1}} \frac{q^{\frac{1}{2} \sum_{i=1}^{m-1} k_{i}\left(k_{i}+1\right)}}{1+q^{|k|-s}},
$$

2000 Mathematics Subject Classification. 11F22, 17B67.

The authors thank the NSF, and the second author thanks the Manasse family, and the Hilldale Foundation for their support. 
where $|k|:=\sum_{i=1}^{m-1} k_{i}$ and

$$
\phi(q):=\prod_{n=1}^{\infty}\left(1-q^{n}\right)
$$

To emphasize the fact that we are working directly with Fourier expansions, we define a related series $K_{r, s}(\tau)$, where $q:=e^{2 \pi i \tau}$ and $\tau \in \mathbb{H}$. If $r \geq 1$ and $s \in \mathbb{Z}$, then let

$$
K_{r, s}(\tau):=q^{-\frac{s}{2}} \frac{\eta^{2}(2 \tau)}{\eta^{r+3}(\tau)} \sum_{k=\left(k_{1}, \ldots, k_{r}\right) \in \mathbb{Z}^{r}} \frac{q^{\frac{1}{2} \sum_{i=1}^{r} k_{i}\left(k_{i}+1\right)}}{1+q^{\sum_{i=1}^{r} k_{i}-s}},
$$

where $\eta(\tau):=q^{\frac{1}{24}} \prod_{n=1}^{\infty}\left(1-q^{n}\right)$ is Dedekind's weight $1 / 2$ modular form. Since we have that

$$
K_{m-1, s}(\tau)=\frac{q^{\frac{1}{12}-\frac{m}{24}}}{2} \operatorname{tr}_{L\left(\Lambda_{(s)}\right)} q^{L_{0}}
$$

it suffices to relate the $K_{r, s}(\tau)$ to weight 0 nonholomorphic modular forms, the nonholomorphic modular functions.

To this end, for $\tau \in \mathbb{H}$ and $u \in \mathbb{C}$, let $c:=\operatorname{Im}(u) / \operatorname{Im}(\tau)$, and define the real analytic function $R(u ; \tau)$ by

$$
R(u ; \tau):=\sum_{\nu \in \mathbb{Z}+\frac{1}{2}}(-1)^{\nu-\frac{1}{2}}\{\operatorname{sgn}(\nu)-E((\nu+c) \sqrt{2 \operatorname{Im}(\tau)})\} e^{-2 \pi i \nu u} q^{-\nu^{2} / 2},
$$

where

$$
E(x):=2 \int_{0}^{x} e^{-\pi u^{2}} d u=\operatorname{sgn}(x)\left(1-\beta\left(x^{2}\right)\right),
$$

and $\beta(x):=\int_{x}^{\infty} u^{-\frac{1}{2}} e^{-\pi u} d u$. For integers $a, b, c$ and $d$, with $c d>0$, we define the weight $1 / 2$ theta function

$$
\vartheta(a, b, c, d ; \tau):=\sum_{n \in \mathbb{Z}} q^{\frac{c d}{2}\left(n+\frac{a}{c}+\frac{b}{d}\right)^{2}} .
$$

These theta functions are related to the classical theta function

$$
\vartheta(u ; \tau):=\sum_{\nu \in \mathbb{Z}+\frac{1}{2}} e^{\pi i \nu^{2} \tau+2 \pi i \nu\left(u+\frac{1}{2}\right)}
$$

by the identity

$$
\vartheta(a, b, c, d ; \tau)=q^{\frac{c d}{2}\left(\frac{a}{c}+\frac{b}{d}-\frac{1}{2}\right)^{2}} \vartheta\left(\left(a d+b c-\frac{c d}{2}\right) \tau-\frac{1}{2} ; c d \tau\right) .
$$

For each $r \geq 1$, we define the finite cone $D_{r}$ in $\mathbb{Z}^{r}$

$$
D_{r}:=\left\{d=\left(d_{1}, d_{2}, d_{3}, \ldots, d_{r}\right) \in \mathbb{Z}^{r}: 0 \leq d_{i} \leq i-1 \text { for each } 1 \leq i \leq r\right\} .
$$

For each $d=\left(d_{1}, d_{2}, d_{3}, \ldots, d_{r}\right) \in D_{r}$, we define

$$
\widetilde{\vartheta}_{d}(\tau):=\prod_{i=1}^{r-1} \vartheta\left(d_{i},-d_{i+1}, i, i+1 ; \tau\right) .
$$


Then we define the weight $-1 / 2$ modular form $\Theta_{r}(\tau)$ by

$$
\Theta_{r}(\tau):=\frac{\eta(2 \tau)^{2}}{\eta(\tau)^{r+3}} \cdot \sum_{d=\left(d_{1}, \ldots, d_{r}\right) \in D_{r}} \widetilde{\vartheta}_{d}(\tau) \cdot q^{\frac{d_{r}^{2}}{2 r}} \vartheta\left(\frac{1}{2}-d_{r} \tau ; r \tau\right) .
$$

Remark. For $r=1$ notice that $D_{1}:=\left\{d=\left(d_{1}\right)=(0)\right\}$, and that $\widetilde{\vartheta}_{d}(\tau):=1$ since empty products are taken to be 1 . Therefore, it follows that

$$
\Theta_{1}(\tau)=\frac{\eta(2 \tau)^{2}}{\eta(\tau)^{4}} \cdot \vartheta\left(\frac{1}{2} ; \tau\right)=-2 \cdot \frac{\eta(2 \tau)^{4}}{\eta(\tau)^{5}}
$$

This last equality follows from the classical identity that $\vartheta\left(\frac{1}{2}, \tau\right)=-2 \cdot \frac{\eta(2 \tau)^{2}}{\eta(\tau)}$. The referee and Sander Zwegers have pointed out more generally that

$$
\Theta_{r}(\tau)=-2^{r} \frac{\eta(2 \tau)^{2 r+2}}{\eta(\tau)^{2 r+3}}
$$

This identity can be proven using the argument in the proof of Theorem 2.1 below. One repeats the transformations in the proof with

$$
\sum_{k=\left(k_{1}, \ldots, k_{r}\right) \in \mathbb{Z}^{r}} q^{\frac{1}{2} \sum_{i=1}^{r} k_{i}\left(k_{i}+1\right)}=\left(\sum_{k \in \mathbb{Z}} q^{\frac{1}{2} k(k+1)}\right)^{r}=2^{r} q^{-\frac{r}{8}} \cdot \frac{\eta(2 \tau)^{2 r}}{\eta(\tau)^{r}}
$$

instead of $L_{r, s}(\tau)$. One then obtains a $q$-series which equals

$$
-q^{-\frac{r}{8}} \sum_{d \in D_{r}} q^{\frac{d_{r}^{2}}{2 r}} \vartheta\left(\frac{1}{2}-d_{r} \tau ; r \tau\right) \widetilde{\vartheta}_{d}(\tau)=-q^{-\frac{r}{8}} \cdot \frac{\eta(\tau)^{r+3}}{\eta(2 \tau)^{2}} \cdot \Theta_{r}(\tau) .
$$

Using $R(u ; \tau), \Theta_{r}(\tau)$ and $K_{r, s}(\tau)$, we define

$$
\widehat{K}_{r, s}(\tau):=q^{-\frac{s^{2}}{2 r}} K_{r, s}(\tau)+\frac{1}{2} q^{-\frac{s^{2}}{2 r}} \Theta_{r}(\tau) R(-s \tau ; r \tau) .
$$

Theorem 1.1. If $r \geq 1$ and $s \in \mathbb{Z}$, then $\widehat{K}_{r, s}(\tau)$ is a nonholomorphic modular function.

Four remarks

1) In view of the fact that $R(-s \tau ; r \tau)$ is a nonholomorphic real analytic function on $\mathbb{H}$, we refer to $\Theta_{r}(\tau) R(-s \tau ; r \tau)$ as the "nonholomorphic part" of $\widehat{K}_{r, s}(\tau)$. We refer to $K_{r, s}(\tau)$ as its "holomorphic part". Consequently, we find that the Kac-Wakimoto characters $\operatorname{tr}_{L\left(\Lambda_{(s)}\right)} q^{L_{0}}$ are, up to powers of $q$, the holomorphic parts of nonholomorphic modular functions. Strictly speaking, this notion is only correct if $s=0$, since in other cases the terms we add to obtain an automorphic form also have holomorphic contributions.

2) By the remark after (1.6), we see that $\Theta_{r}(\tau)$ is nonvanishing on the upper-half of the complex plane. Therefore, it follows that

$$
\frac{\widehat{K}_{r, s}(\tau)}{\Theta_{r}(\tau)}
$$

is a weight $1 / 2$ harmonic Maass form. 
3) It is now straightforward to deduce the modular transformations for $\widehat{K}_{r, s}(\tau)$ for every element in $\mathrm{SL}_{2}(\mathbb{Z})$ using the results in $\S 2$ and classical transformation laws for Dedekind's eta-function and the $\vartheta_{a, b, c, d}(\tau)$. For brevity we do not give the tedious details here.

4) Although we consider $\operatorname{tr}_{L\left(\Lambda_{(s)}\right)} q^{L_{0}}$ for simplicity, we note that the general character $\operatorname{ch} L\left(\Lambda_{(s)}\right)$ for $s \ell(m, 1)^{\wedge}$ may also be expressed in terms of nonholomorphic Jacobi-type forms. Using (4.2) of [4] and the $q$-series manipulations in $\S 2$ (decorated to accommodate the $z_{i}$ factors), one obtains an expression involving classical theta functions and the $\widehat{\mu}$ function of Zwegers. Theorem 2.4 then gives the result.

\section{Proof of Theorem 1.1}

Here we prove Theorem 1.1 by combining some $q$-series identities with work from Zwegers's thesis. We begin with some $q$-series identities.

2.1. $q$-series manipulations. To prove Theorem 1.1, it is enough to consider

$$
L_{r, s}(\tau):=\sum_{k=\left(k_{1}, \ldots, k_{r}\right) \in \mathbb{Z}^{r}} \frac{q^{\frac{1}{2} \sum_{i} k_{i}\left(k_{i}+1\right)}}{1+q^{-s+\sum_{i} k_{i}}} .
$$

We define

$$
\mu_{d, r, s}(\tau):=q^{-\frac{d}{2}+\frac{d^{2}}{2 r}} \sum_{n \in \mathbb{Z}} \frac{q^{\frac{r n}{2}(n+1)-d n}}{1+q^{r n-d-s}} .
$$

Theorem 2.1. We have

$$
L_{r, s}(\tau)=\sum_{d=\left(d_{1}, \cdots, d_{r}\right) \in D_{r}} \widetilde{\vartheta}_{d}(\tau) \mu_{d_{r}, r, s}(\tau)
$$

For the proof of Theorem 2.1, we require the following lemma.

Lemma 2.2. For $j \geq 2, M \in \mathbb{R}, k_{j} \in \mathbb{Z}$, and $d_{j} \in \mathbb{R}$, we define

$$
a_{j}:=\frac{j(j+1)}{2}\left(k_{j}+\frac{d_{j}}{j}-\frac{1}{j+1}\left(k_{j+1}-M\right)\right)^{2}-\frac{j}{2(j+1)}\left(k_{j+1}-M\right)^{2}+\left(k_{j+1}^{2}-M k_{j+1}\right) .
$$

Then under the change of variables

$$
\begin{aligned}
k_{j+1} & \mapsto(j+1) k_{j+1}+d_{j+1}+M, \\
k_{j} & \mapsto k_{j}+k_{j+1}
\end{aligned}
$$

$a_{j}$ becomes

$$
\frac{j(j+1)}{2}\left(k_{j}+\frac{d_{j}}{j}-\frac{d_{j+1}}{j+1}\right)^{2}+\frac{(j+1)(j+2)}{2}\left(k_{j+1}+\frac{d_{j+1}}{j+1}+\frac{M}{j+2}\right)^{2}-\frac{M^{2}(j+1)}{2(j+2)} .
$$

Proof. Under the transformation the $a_{j}$ become

$\frac{j(j+1)}{2}\left(k_{j}+\frac{d_{j}}{j}-\frac{d_{j+1}}{j+1}\right)^{2}-\frac{j}{2(j+1)}\left((j+1) k_{j+1}+d_{j+1}\right)^{2}+\left((j+1) k_{j+1}+\frac{M}{2}+d_{j+1}\right)^{2}-\frac{M^{2}}{4}$. 
The first term is the first term of (2.9). We simplify the remaining terms as

$$
\begin{aligned}
\frac{(j+1)(j+2)}{2} k_{j+1}^{2}+(j+2) d_{j+1} & k_{j+1}+(j+1) M k_{j+1}+\frac{j+2}{2(j+1)} d_{j+1}^{2}+M d_{j+1} \\
& =\frac{(j+1)(j+2)}{2}\left(k_{j+1}+\frac{d_{j+1}}{j+1}+\frac{M}{j+2}\right)^{2}-\frac{M^{2}(j+1)}{2(j+2)}
\end{aligned}
$$

which gives the claim.

Proof of Theorem 2.1. The claim is simple in the case that $r=1$. Therefore, we assume that $r \geq 2$. We make the change of variables $k_{r} \mapsto k_{r}-\sum_{i=1}^{r-1} k_{i}$. This transforms the series into

$$
L_{r, s}(\tau)=\sum_{k=\left(k_{1}, \ldots, k_{r}\right) \in \mathbb{Z}^{r}} \frac{q^{\frac{1}{2} k_{r}\left(k_{r}+1\right)+\sum_{i=1}^{r-1}\left(k_{i}^{2}-k_{r} k_{i}+k_{i} \sum_{\ell=i+1}^{r-1} k_{\ell}\right)}}{1+q^{-s+k_{r}}} .
$$

We now make several changes of variables which do not change the denominator of this series. We start with the exponent

$$
\frac{1}{2} k_{r}\left(k_{r}+1\right)+\sum_{i=1}^{r-1}\left(k_{i}^{2}-k_{r} k_{i}+k_{i} \sum_{\ell=i+1}^{r-1} k_{\ell}\right) .
$$

Firstly we let for $r>2$

$$
\begin{aligned}
& k_{2} \mapsto 2 k_{2}+k_{r}-\sum_{\ell=3}^{r-1} k_{\ell}+d_{2}, \\
& k_{1} \mapsto k_{1}-k_{2},
\end{aligned}
$$

where $0 \leq d_{2} \leq 1$. The only terms that are changed by this change of variables are

$$
\begin{aligned}
& \left(k_{2}^{2}-k_{r} k_{2}+k_{2} \sum_{\ell=3}^{r-1} k_{\ell}\right)+\left(k_{1}^{2}-k_{r} k_{1}+k_{1} \sum_{\ell=2}^{r-1} k_{\ell}\right) \\
= & \left(k_{2}+\frac{K}{2}\right)^{2}-\frac{K^{2}}{4}+\left(k_{1}+\frac{1}{2}\left(k_{2}+K\right)\right)^{2}-\frac{1}{4}\left(k_{2}+K\right)^{2},
\end{aligned}
$$

where $K:=-k_{r}+\sum_{\ell=3}^{r-1} k_{\ell}$. Now the change of variables (2.10) yields the expression

$$
\left(2 k_{2}-\frac{K}{2}+d_{2}\right)^{2}-\frac{K^{2}}{4}+\left(k_{1}+\frac{d_{2}}{2}\right)^{2}-\frac{1}{4}\left(2 k_{2}+d_{2}\right)^{2}=\left(k_{1}+\frac{d_{2}}{2}\right)^{2}+3\left(k_{2}+\frac{d_{2}}{2}-\frac{K}{3}\right)^{2}-\frac{K^{2}}{3} \text {. }
$$

Thus the whole exponent equals

$$
\begin{aligned}
\frac{1}{2} k_{r}\left(k_{r}+1\right)+ & \sum_{i=3}^{r-1}\left(k_{i}^{2}-k_{r} k_{i}+k_{i} \sum_{l=i+1}^{r-1} k_{l}\right) \\
& +\left(k_{1}+\frac{d_{2}}{2}\right)^{2}+3\left(k_{2}+\frac{d_{2}}{2}+\frac{1}{3}\left(k_{r}-\sum_{j=3}^{r-1} k_{j}\right)\right)^{2}-\frac{1}{3}\left(k_{r}-\sum_{j=3}^{r-1} k_{j}\right)^{2} .
\end{aligned}
$$


We now apply successively for $2 \leq j \leq r-2$ the transformations

$$
\begin{aligned}
k_{j+1} & \mapsto(j+1) k_{j+1}+d_{j+1}+k_{r}-\sum_{\ell=j+2}^{r-1} k_{\ell}, \\
k_{j} & \mapsto k_{j}+k_{j+1},
\end{aligned}
$$

with $0 \leq d_{j+1} \leq j$. We claim that after the $j$ th step the exponent equals

$$
\begin{aligned}
& \text { (2.11) } \frac{k_{r}}{2}\left(k_{r}+1\right)+\sum_{i=j+2}^{r-1}\left(k_{i}^{2}-k_{r} k_{i}+k_{i} \sum_{\ell=i+1}^{r-1} k_{\ell}\right) \\
& +\frac{(j+1)(j+2)}{2}\left(k_{j+1}+\frac{d_{j+1}}{j+1}-\frac{1}{j+2}\left(-k_{r}+\sum_{\ell=j+2}^{r-1} k_{\ell}\right)\right)^{2}-\frac{j+1}{2(j+2)}\left(-k_{r}+\sum_{\ell=j+2}^{r-1} k_{\ell}\right)^{2} \\
& +\frac{j(j+1)}{2}\left(k_{j}+\frac{d_{j}}{j}-\frac{d_{j+1}}{j+1}\right)^{2}+\cdots+3\left(k_{2}+\frac{d_{2}}{2}-\frac{d_{3}}{3}\right)^{2}+\left(k_{1}+\frac{d_{2}}{2}\right)^{2} .
\end{aligned}
$$

To see this, we use induction over $j$ and Lemma 2.2. We first consider the case $j=2$. The only terms influenced by the change of variables are

$$
\left(k_{3}^{2}-k_{r} k_{3}+k_{3} \sum_{\ell=4}^{r-1} k_{\ell}\right)+3\left(k_{2}+\frac{d_{2}}{2}+\frac{1}{3}\left(k_{r}-\sum_{\ell=3}^{r-1} k_{\ell}\right)\right)^{2}-\frac{1}{3}\left(k_{r}-\sum_{\ell=3}^{r-1} k_{\ell}\right)^{2} .
$$

Equation (2.11) for $j=2$ follows from Lemma 2.2 with $j=2$ and $M=k_{r}-\sum_{\ell=4}^{r-1} k_{\ell}$. Next we assume that (2.11) is true for $j$ and we show that it holds also for $j+1$. The only terms that play a role in the change of variables are

$$
\begin{aligned}
& \left(k_{j+2}^{2}-k_{r} k_{j+2}+k_{j+2} \sum_{\ell=j+3}^{r-1} k_{\ell}\right) \\
+ & \frac{(j+1)(j+2)}{2}\left(k_{j+1}+\frac{d_{j+1}}{j+1}+\frac{1}{j+2}\left(k_{r}-\sum_{\ell=j+2}^{r-1} k_{\ell}\right)\right)^{2}-\frac{j+1}{2(j+2)}\left(-k_{r}+\sum_{\ell=j+2}^{r-1} k_{\ell}\right)^{2} .
\end{aligned}
$$

The claim now follows from Lemma 2.2 with $j+1$ instead of $j$ and $M=k_{r}-\sum_{\ell=j+3}^{r-1} k_{\ell}$.

Thus we have after all the above described transformations the exponent

$$
\begin{aligned}
\frac{k_{r}}{2}\left(k_{r}+1\right)+\frac{r(r-1)}{2}\left(k_{r-1}+\frac{d_{r-1}}{r-1}\right. & \left.+\frac{k_{r}}{r}\right)^{2}-\frac{(r-1)}{2 r} k_{r}^{2} \\
& +\left(k_{1}+\frac{d_{2}}{2}\right)^{2}+\frac{1}{2} \sum_{\ell=2}^{r-2} \ell(\ell+1)\left(k_{\ell}+\frac{d_{\ell}}{\ell}-\frac{d_{\ell+1}}{\ell+1}\right)^{2} .
\end{aligned}
$$

We make the change of variables for $r \geq 2$

$$
\begin{aligned}
k_{r} & \mapsto r k_{r}-d_{r}, \\
k_{r-1} & \mapsto k_{r-1}-k_{r} .
\end{aligned}
$$


Only the following terms play a role:

$$
\begin{aligned}
\frac{k_{r}}{2}\left(k_{r}+1\right)+\frac{r(r-1)}{2}\left(k_{r-1}+\right. & \left.\frac{d_{r-1}}{r-1}+\frac{k_{r}}{r}\right)^{2}-\frac{(r-1)}{2 r} k_{r}^{2} \\
& =\frac{1}{2 r}\left(k_{r}+\frac{r}{2}\right)^{2}-\frac{r}{8}+\frac{(r-1) r}{2}\left(k_{r-1}+\frac{d_{r-1}}{r-1}+\frac{k_{r}}{r}\right)^{2} .
\end{aligned}
$$

Making the above described change of variables transforms this term into

$$
\frac{(r-1) r}{2}\left(k_{r-1}+\frac{d_{r-1}}{r-1}-\frac{d_{r}}{r}\right)^{2}+\frac{r k_{r}^{2}}{2}-d_{r} k_{r}+\frac{r k_{r}}{2}+\frac{d_{r}^{2}}{2 r}-\frac{d_{r}}{2} .
$$

This yields that $L_{r, s}(\tau)$ equals

$$
\sum_{0 \leq d_{i} \leq i-1} \sum_{k_{i} \in \mathbb{Z}} \frac{q^{\frac{r k_{r}^{2}}{2}-d_{r} k_{r}+\frac{r k_{r}}{2}+\frac{d_{r}^{2}}{2 r}-\frac{d_{r}}{2}+\frac{1}{2} \sum_{\ell=2}^{r-1} \ell(\ell+1)\left(k_{\ell}+\frac{d_{\ell}}{\ell}-\frac{d_{\ell+1}}{\ell+1}\right)^{2}+\left(k_{1}+\frac{d_{2}}{2}\right)^{2}}}{1+q^{r k_{r}-s-d_{r}}} .
$$

The theorem now follows easily from the definition of $\widetilde{\vartheta}_{d}(\tau)$ and $\mu_{d_{r}, r, s}(\tau)$.

2.2. Zwegers's $\mu$-function and the proof of Theorem 1.1. In his thesis, Zwegers constructed weight $1 / 2$ harmonic Maass forms by making use of the transformation properties of Lerch sums. We briefly recall some of his important results.

For $\tau \in \mathbb{H}, u, v \in \mathbb{C} \backslash(\mathbb{Z} \tau+\mathbb{Z})$, Zwegers defined the function

$$
\mu(u, v ; \tau):=\frac{z^{1 / 2}}{\vartheta(v ; \tau)} \cdot \sum_{n \in \mathbb{Z}} \frac{(-w)^{n} q^{n(n+1) / 2}}{1-z q^{n}},
$$

where $z:=e^{2 \pi i u}, w:=e^{2 \pi i v}, q:=e^{2 \pi i \tau}$. Zwegers (see $\S 1.3$ of [12]) proved that $\mu(u, v ; \tau)$ satisfies the following transformations.

Lemma 2.3. Assuming the notation above, we have that

$$
\begin{aligned}
\mu(u, v ; \tau) & =\mu(v, u ; \tau), \\
\mu(u+1, v ; \tau) & =-\mu(u, v ; \tau), \\
z^{-1} w q^{-\frac{1}{2}} \mu(u+\tau, v ; \tau) & =-\mu(u, v ; \tau)-i z^{-\frac{1}{2}} w^{\frac{1}{2}} q^{-\frac{1}{8}}, \\
\mu(u, v ; \tau+1) & =\zeta_{8}^{-1} \mu(u, v ; \tau) \quad\left(\zeta_{N}:=e^{2 \pi i / N}\right) \\
(\tau / i)^{-\frac{1}{2}} e^{\pi i(u-v)^{2} / \tau} \mu\left(\frac{u}{\tau}, \frac{v}{\tau} ;-\frac{1}{\tau}\right) & =-\mu(u, v ; \tau)+\frac{1}{2 i} h(u-v ; \tau),
\end{aligned}
$$

where

$$
h(z ; \tau):=\int_{-\infty}^{\infty} \frac{e^{\pi i x^{2} \tau-2 \pi x z} d x}{\cosh \pi x} .
$$

Lemma 2.3 shows that $\mu(u, v ; \tau)$ is nearly a weight $1 / 2$ Jacobi form, where $\tau$ is the modular variable. Zwegers then used $\mu$ to construct harmonic Maass forms. Using $\mu$ and $R$, Zwegers defined the real analytic function

$$
\widehat{\mu}(u, v ; \tau):=\mu(u, v ; \tau)+\frac{i}{2} R(u-v ; \tau) .
$$


At torsion points, this function specializes to give weight $1 / 2$ harmonic Maass forms. This is apparent from the following theorem.

Theorem 2.4. Assuming the notation and hypotheses above, we have that

$$
\begin{aligned}
\widehat{\mu}(u, v ; \tau) & =\widehat{\mu}(v, u ; \tau), \\
\widehat{\mu}(u+1, v ; \tau) & =z^{-1} w q^{-\frac{1}{2}} \widehat{\mu}(u+\tau, v ; \tau)=-\widehat{\mu}(u, v ; \tau), \\
\zeta_{8} \widehat{\mu}(u, v ; \tau+1) & =-(\tau / i)^{-\frac{1}{2}} e^{\pi i(u-v)^{2} / \tau} \widehat{\mu}\left(\frac{u}{\tau}, \frac{v}{\tau} ;-\frac{1}{\tau}\right)=\widehat{\mu}(u, v ; \tau) .
\end{aligned}
$$

Moreover, if $A=\left(\begin{array}{ll}\alpha & \beta \\ \gamma & \delta\end{array}\right) \in \mathrm{SL}_{2}(\mathbb{Z})$, then

$$
\widehat{\mu}\left(\frac{u}{\gamma \tau+\delta}, \frac{v}{\gamma \tau+\delta} ; \frac{\alpha \tau+\beta}{\gamma \tau+\delta}\right)=\chi(A)^{-3}(\gamma \tau+\delta)^{\frac{1}{2}} e^{-\pi i \gamma(u-v)^{2} /(\gamma \tau+\delta)} \cdot \widehat{\mu}(u, v ; \tau),
$$

where $\chi(A):=\eta(A \tau) /\left((\gamma \tau+\delta)^{\frac{1}{2}} \eta(\tau)\right)$.

Proof of Theorem 1.1. For the proof, we require the fact (see Theorem 1.3 of [1]) that if $\Phi(\tau, z)$ is a Jacobi form of weight $k$ and index $m$, then for $\lambda, \mu \in \mathbb{Q}, q^{m \lambda^{2}} \Phi(\tau, \lambda \tau+\mu)$ is a modular form of weight $k$ on some congruence subgroup. Using that $\vartheta(u ; \tau)$ is a Jacobi form of weight $\frac{1}{2}$ and index $\frac{1}{2}$ then yields that $\vartheta(a, b, c, d ; \tau)$ and thus $\widetilde{\vartheta}$ and $\Theta_{r}$ are modular forms.

Next observe that

$$
q^{-\frac{s}{2}} \mu_{d_{r}, r, s}(\tau)=-i q^{\frac{d_{r}^{2}}{2 r}} \vartheta\left(\frac{1}{2}-d_{r} \tau ; r \tau\right) \mu\left(\frac{1}{2}-\left(s+d_{r}\right) \tau,-d_{r} \tau+\frac{1}{2} ; r \tau\right) .
$$

For brevity we consider the $s=0$ case. In the general case, one argues in an analogous way after first employing the elliptic transformation laws in Lemma 2.3.

Using Theorem 2.4, we see that for $\alpha, \beta, \gamma, \delta \in \mathbb{Q}$ with $-\frac{1}{2}<\gamma-\alpha<\frac{1}{2}$ we have

$$
q^{-\frac{1}{2}(\gamma-\alpha)^{2}} \mu(\gamma \tau+\delta, \alpha \tau+\beta ; \tau)
$$

is the holomorphic part of a (nonholomorphic) modular form of weight $\frac{1}{2}$. Thus each of the functions

$$
\mu_{d_{r}, r, 0}(\tau) \widetilde{\vartheta}_{d}(\tau)
$$

are holomorphic parts of nonholomorphic modular forms whose non-holomorphic part is given by

$$
\frac{1}{2} q^{\frac{d_{r}^{2}}{2 r}} \widetilde{\vartheta}_{d}(\tau) \vartheta\left(\frac{1}{2}-d_{r} \tau ; r \tau\right) R(0 ; r \tau)
$$

Note that the only nonholomorphic contribution is $R(0 ; r \tau)$ which is independent of $d$. The theorem now follows directly from Theorem 2.1.

2.3. Some examples. Here we consider two examples of these results.

Example. We have seen that $\Theta_{1}(\tau)=-2 \eta(2 \tau)^{4} / \eta(\tau)^{5}$. As a consequence, Theorem 1.1 gives the following nonholomorphic modular function

$$
\widehat{K}_{1, s}(\tau)=q^{-\frac{s^{2}}{2}} K_{1, s}(\tau)-q^{-\frac{s^{2}}{2}} \frac{\eta(2 \tau)^{4}}{\eta(\tau)^{5}} R(-s \tau ; \tau)
$$


Example. For $r=2$, we have that $\Theta_{2}(\tau)=-4 \eta(2 \tau)^{6} / \eta(\tau)^{7}$. Theorem 1.1 gives the following nonholomorphic modular function

$$
\widehat{K}_{2, s}(\tau)=q^{-\frac{s^{2}}{4}} K_{2, s}(\tau)-2 q^{-\frac{s^{2}}{4}} \frac{\eta(2 \tau)^{6}}{\eta(\tau)^{7}} R(-s \tau ; 2 \tau) .
$$

\section{ACKNOWLEDGEMENTS}

The second author thanks Victor Kac for stimulating discussions on subjects related to the subject of this paper, and both authors thank Sander Zwegers for pointing out typographical errors in an earlier version of this paper. Moreover they thank the referee for helpful comments.

\section{REFERENCES}

[1] M. Eichler and D. Zagier, The theory of Jacobi forms, Progress in Mathematics 55. Birkhäuser Boston, Inc., Boston, MA, 1985. v+148 pp.

[2] V. G. Kac and D. H. Peterson, Infinite-dimensional Lie algebras, theta functions, and modular forms, Adv. Math. 53 (1984), pages 125-264.

[3] V. G. Kac and M. Wakimoto, Integrable highest weight modules over affine superalgebras and number theory, Lie theory and geometry, Progr. Math. 123, Birkhäuser, Boston, 1994, pages 415-456.

[4] V. G. Kac and M. Wakimoto, Integrable highest weight modules over affine superalgebras and Appell's function, Comm. Math. Phys. 215 (2001), no. 3, pages 631-682.

[5] S. Milne, New infinite families of exact sums of squares formulas, Jacobi elliptic functions, and Ramanujan's tau function, Proc. Nat. Acad. Sci. U.S.A. 93 (1996), no. 26, pages 15004-15008.

[6] S. Milne, Infinite families of exact sums of squares formulas, Jacobi elliptic functions, continued fractions, and Schur functions, Ramanujan J. 6 (2002), no. 1, pages 7-149.

[7] K. Ono, Representations of integers as sums of squares, J. Number Theory 95 (2002), no. 2, pages $253-258$.

[8] K. Ono, Unearthing the visions of a master: harmonic Maass forms and number theory, Harvard-MIT Current Developments in Mathematics 2008, International Press, accepted for publication.

[9] M. Wakimoto, Representation theory of affine superalgebras at the critical level, Proc. Intl. Congr. Math., Vol II (Berlin, 1998) Doc. Math., Extra Vol. II, pages 605-614.

[10] D. Zagier, A proof of the Kac-Wakimoto affine denominator formula for the strange series, Math. Res. Lett. 7 (2000), no. 5-6, pages 597-604.

[11] D. Zagier, Ramanujan's mock theta functions and their applications [d'aprés Zwegers and BringmannOno] (2006), Séminaire Bourbaki, no 986.

[12] S. Zwegers, Mock theta functions, Ph.D. Thesis (Advisor: D. Zagier), Universiteit Utrecht, 2002.

Mathematisches Institut, Universität Köln, Weyertal 86-90, D-50931 Köln, Germany

E-mail address: kbringma@math.uni-koeln.de

Department of Mathematics, University of Wisconsin, Madison, Wisconsin 53706

E-mail address: ono@math.wisc.edu 